à-dire les intérêts des producleurs d'énergie plutòt que ceux des consommaleurs : ni les Chambres de Commerce, ni les collectivités locales chargées de Services publics tributaires de l'énergie, n'y sont représentés.

On doit admettre, cependant, qu'en matière d'énergie, comme en matière de transports, les solutions conformes à l'intérêt général ne peuvent être dégagées que par des organismes où prédominent à côté de parlementaires ét de foncliomnaires, ne connaissant les besoins que de seconde main, par des rapports, les représentants des consommateurs d'énergie et non ceux des producteurs.

V. - En résumé, le bénéfice que les Services publics sonl appelés a retirer du nouveau projel ae loi va dependre, en grande partıe du concours que l titat et les collectivités rocales trouveront aupres des socretés réglonales de transporl el de distribution a énergie électrique.

Jc me léserve d'aborider, daus une prochaine étude, l'examen des moyens qui se présentenl de "coordonner" les concessions a'usines hyarauliques, prévues par le projet de toi, avec les divers lypes de concessions de distributions d'énergie prévus, sans qu'il en ait été fait jusqu'icı d'application systématıque, par les arrêtés des $\mathrm{r}_{7}$ mai rgos', 20 aoúl 1908 , 3o noveinbre 1909 , rendus en exécution de la loi du 15 juin $1906 . J$ fas seulement remarquer que le cahier des charśs-type, approuvé par le dernier arrêté, vise précisémenl les concessions de dıstribution d'énergie aux Services publics, c'est-à-dire le plus important de tous les bervices publics, tributaires de la houlle blanche, puisque, seul, il peut assurer le bon fonctionnemenl des autres.

De ce premier examen du projet de loi; je tirerai ceite seule conclusion que les collectivités, chargées de pourvoir aux besoins d'énergie des Services publics, trouveront, dans la législation nouvelle, des moyens d'action précieux, à la condition de se grouper et de s entendre pour obtenir, dans la mesure qui convient, le concours indispensable des Société de transport et de distribution d'énergie électrique.

Par la création d'Offices d'énergie, analog ues aux Uffices des Transports, et constitués par l'initiative des Chambres de Commerce, qui paraissent qualifiées pour cela, les intérêts des Services publics et de l'Industrie ; d'une façọn plus générale, ceux des producleurs el des consommateurs d'énergie, seront 'équitablement conciliés. Les ententes préconisées par l'exposé des motifs seront préparées. La tấche du Comité consultatif des Forces hydrauliques sera facilitée.

Cetle conclusion n'entraîne comme conséquence qu'une seule modilication de texte visant l'art. 29 qui règle dans un cadre peut-être trop rigide', ta composition du Comité consultatif des Forces hydrauliques. Il serait bon que ce Comité pùt être, comme le Comité consultatif des chemins de ler, renforcé par l'adjonction de personnalités régionales compétentes, représentant les Offices régionaux d'énergie.

VI. - Le vote de la loi par le Parlement et surtout la préparation des règlements multiples, si délicats, qu'elle prévoit, vont sûrement exiger de longs délais. Il suffit,pour s'en faire une idée, de con'stater que le régime des concessions, olganisé pour les distributions d'énergie par l'arsenal des règlements qu'a fait, éclore la loi du 15 juin $x g o 6$, 'n'a pas encore normalement fonctionné.

D'autre part, la délimitation des centres économiques régionaux où seront appelés à fonctionner les "Offices d'énergie $n$, sous leur forme définitive, ne peut être l'œuvre d'un jour.

Les besoins sont impérieux cependant ; il serait criminel d'attendre pour les satisfaire que fût achevée, avec tous les 'détails qu'elle comporte, la réforme législative en cours. II faut se servir des organismes qui fonctionnent et des lois existantes, spécialement des organismes et des lois que la guerre a fait naître.

Les Comités consultatifs d'action économiques créés par le décret du 20 octobre 1915 , se sont préoccupés déjà, dans beaucoup de régions, des difficullés si graves qui résultent du manque d'énergie. Ils sont qualifiés pour jouer provisoirement le rôle des Offices d'énergie, en altendant que ceux-ci les remplacent. Rien ne les empêche de coordonner leur action d'une région à l'autre, en la rendant ainsi plus efficace.

Les lois exislantes permeltent, sans aucun doute possible. de déclarer d'utilité publique avec urgence el d'entreprendre de suite, sur des terrains occupés par réquisition, la construction d'usines hydrauliques inléressant la défense nalionale, destinées plus tard à alimenter les Services publias de l'Etal, des Départements, des Villes, etc...

Sans perdre un instant, il faul user de ces moyens el mellre en jeu ces organismes. Les administrations de l'Etal ou les administraitons locales qui plus tard, après salisfaction des besoins de guerre el d'après-guerre, auront à utiliser: les usines pour les Services publics de loules calégories: chomins de fer, irrigation, distributions publiques d'énergic ćlectrique, etc., doivent concourir, par des ententes avec les administration de guerre, à l'aménagenent des Forces hydrauliques dont le pays a un si grand besoin. Lorsque, dans ces cntenles, n'interviendront que des collectivités, il sera facile de sauvegarder d'une façon sủre les intérêts généraux, en réservant, au besoin, l'applicalion de la loi projetée el des règlements à intervenir.

Des tentatives de toutes sorles, dans toutes les directions, utilisant des concours, faciles à obtenir à l'heure actuelle, auront l'avantage de permettre d'essayer la loi avant qu'ello ne soit arrêtée dans ses détails, cn contribuant ainsi à lui assurer le fonctionnement le plus souple el le plus conforme à l'intérêt général.

René Tavernier.

Inspecteur général des Ponts el Chaussées, en retraile.

\section{L'AMÉNAGEMENT DU RHONE} ÉTAT DE LA QUESTION

\section{CONSIDERATIONS RETROSPECTIVES}

Avant la Guerre, quand on parlail, dans les sphères administratives, comme dans le monde des grandes entreprises, des 500 ou 600 millions que coûtérait l'aménagement rationnel du Rhône pour en faire à la fois la plus productive de nos voies de transports et la plus puissante de nos mines de houille blanche, on renvoyait aux calendes grecques l'examen de toute entreprise susceptible de se solder par uno dépense de cet ordre de grandeur.

L'Administration des Travaux publics doutail de la possibilité des solutions proposées pour la parlie du probleme relative à la Navigation, et n'appréciait pas les moyens que les derniens progrès de l'Hydraulique et de l'Electricité industrielles fournissent au technicien pour la misc en ceuvre de l'énorme quantité d'énergie dynamiquc en puissance dans le cours d'ealu.

Il en était résulté cet état d'espril, presque, général dans les milieux qui possédaient les moyens de réalisation : grand travail répondanl sans doute à une utilité... mais, 6oo rnillions, c'est un chiffre 1...

Or, la Guerre est venue ; par semaine, en or seulement, clle nous coûte plus que cela.

De combien ne nous en aurait-il pas moins coûté s l'emploi de ces millions avait été fait avant?

Rien ne sert maintenant d'épilóguer sur les conséquences de ces faits que leur opposition rend douloureusement stupéfiante. L'apathie qui fut, au ford, la cause de cetle si funeste erreur de jugement, est vaincue : les solutions cnvisagées pour sauver dans le temps voulu l'indépendance in- 
dustrielle de notie Pays, sont abordées avec un esprit nouveau qui lémoigne de décision et de volonté ; l'union de tous les concours réalisèra l'oesuvre léparatrice de l'avenir.

Voulanl que La Houille Blanche ajoute sa "ligne de foree " au flux dense de celles qui vont puissamment grandir le chámp de l'aclivité nationale, nous ouvrons, à nouveau, ses colonnes et plus largement encorè que par le passé, aux auteurs compétents qui voudront bien les consacrer a cetle section parliculièrement importante de l'outillage économique de la Fiance que doit constituer l'aménagement de ses cours d'eau navigables.

Les projets relatifs aux diverses utilisations dont le Rhòne ost susceplible le long de son cours sur le territoire francais, peuvent se classer en deux calégories principales

la première comprend les études ayant pour but l'amélionalion de la navigabilité dans la principale section, de la Mer à Lyon, afin de faire de cette voie l'un des plus imporlants trongons d'une grande ligne de communicalion par eau entre la Méditerranée et la Mer du Nord. Aucun souci bien avoué de lirer parli de la force motrice, susceptible d'être produite par ladaptation à cet effet de certains tràvaux prévus pour la navigation n'est apparue dans ces projels avant les premières éludes publiées sur cette queslion, il y a une dizaine d'années, par M. René Tavernier, alors lingénieur en chef des Ponts et Chaussées (I).

La deuxième catégoric comprend les projels d'usines pour l'utilisation des chutes du Rhône dans son parcours du lac de Geneve à Lyon. Ici, toul est au contraire conçu pour la meilleure adaptation des ouvrages à leur but essenliel : la production de force motrice. Les installations sont néanmoins prévués pour qu'à leurs lravaux de captation de cetle force molrice corresponde un programme d'ouvrages destinés à mettre chaque section de cours d'eas coniprise entre deux usines cn état de devenir un bief de navigation qui constituera l'un des échelons de la voie d'eau remontant de Lyon à Genère.

I a Houlle Blanche, ses plus anciens lecteurs doivent s'en souvenir, a rempli avant la guerre, nombre de ses pages des plus importantes éludes sur les projets d'aménagements des diverses sections du Rhône.

Avant de donner plus de place encore a ces questions dans notre Revue, nous estimons utile de rappeler en un court l'śsumé, la siluation des principaux travaux à l'étude au moment oú la guerro les a interiompus.

\section{LES PROJETS SUR LE RHONE DE LYON A GENEVE}

En ce qui concerne la situation de ces projets, nous ne jouvons mieux faire que tires nos renseignements du comple rendu de l'Assemblée générale du Comité FrancoSuisse du Haut Rhône, tenue le 3 mars 1917 , à la Chambre de Commirce de lyon, sous la présidence de son président, V. Joan Corcner.

Avant d'aller plus Join, nous devons d'abord dire ce qu'est Je Comité Franco-Suisse du Hanl-Rhone. - Il a été constitué deux avant la guerre, sur l'initialive de la Chambre de Commerce française de Geneve pour entreprendre et faciliter l'étude de la navigation entre Iyon et Genève et dans le but de créer une entente entre la Suisse et la France, pour que les travaux d'aménagement soient menés parallèlement de part et d'untre.

Ses Statuls comprennent notamment les dispositions suirantes, qui spécifient bicn son but :

(1) Nous conseillons aux lecteurs que cette question intéresse de se reporter à la conférence faite par M. René TAvernier, à la Soczété d'Economie politique de Lyon, (9t Mars 1908).
Art. 2. - L'Association a pour objet de préparer l'entente internationale nécessaire à la réalisation de la voie navigable de Lyon à Genève par le Rhône ou par des canaux latéraux à ce fleuve.

Elle prend connaissance des projets élablis par les Associalions françaises et suisses poursuivant séparément un bul analogue, coordonne les études et les recherches destiñéés à l'aménagement du Haut-Rhòne sur ce parcours, concilie les intérêts de la captation de l'énergie hydraulique avec les exigences de la navigation el sert d'intermédiaire à ces diverses Associations auprès des Pouvoirs publics des deux Etats riverains.

Art. 4. - La durée de l'Association n'est pas déterminée; le but poursuivi par elle sera considéré comme atteint lorsqu'une entente définitive aura été conclue entre les Gouvernements français et suisse sur l'aménagement du HautRhône, en vue de la création d'une voie de grande navigalion entre Lyon et Genève et lorsque cette voie aura été mise entièrement à exécution. L'Associātion serd alors dissoule.

ART. 5. - Les Sociétaires sont choisis parmi les membres des Associations, des Compagnies, des Municipalités et des Entreprises suivantes.

Association française pour l'Amélioration et la Défense de la Navigation intérieure. - Union des Chambres de Commerce françaises à l'Etranger, aux Colonies et aux Pays de Protectorat. - Association, suisse pour la Navigation du Rhône au Rhin. - Syndicat suisse pour l'Etude de la Voie navigable du Rhône au Rhin. - Chambres de Commerce de Paris, de Lyon, de Marseille el de Bourg. - Compagnie lyonnaise de Navigation et de Remorquage. - Compagnie. générale de Navigation. - Société lyonnaise des Forcés motrices du Rhône. - Villes de Lyon, Genève, Bellegarde et Lausanne. - Usines de Génissiat. - Société dos Grands Travaux de Marseille.

ART. 9. - L'Association admet, en qualité d'adhérents, des personnes ou des Associations qui, moyennant une contribution annuelle, sont tenues au courant de l'activité du Comité et reçoivent les publications relatives à ses travaux.

Le nombre des adhérents est illimité ; le montant de leur: contribution annuelle est fixé par la Direction.

Voici la composition actuelle de son Bureau :

Président : M. J. CoIgnet, Président de la Chambre de Commerce de Lyon.

Vice-Présidents : MM. E. MAzuY, Président de la Chambre de Commerce française de Genève.- M. H. Romreux, ancien Conseiller d'Etat, à Genève.

Secrétaire : M. G. Autran, ingénieur, à Genève.

Secrétaire-Adjoint : M. P. SAvole-Petrtprerne; Président central de l'Association suisse pour la Navigation du Rhône au Rhin.

Trésorier : M. W. Martin, ingénieur, à Lausanne.

Trésorier-Adjoint : M. E. Coü̈Bss, Vice-Président de là: Chambre de Commerce de Bourg.

Le Bureau de ce Comité a tenu une première séance le I2 juîn rgr2, à la Chambre de Commerce française de Genève. Il y fut examiné les diverses questions suivantes:

Le programme d'une Conférence internationale devant avoir lieu entre les ingénieurs de l'Etat, français et suisses, pour examiner les conditions d'installation de l'usine internationale hydro-électrique de Chancy-Pougny,, dont la concession est sollicitée concurremment par un groupe francosuisse et par la Ville de Genève.

Un avant-projet de la Société française des Forces hydrauliques du Rhône, à Bellegarde, en vue de l'établissement d'un canal de navigation entre Génissiat et la perte du Rhône au moyen de deux biefs successifs.

Les plans généraux des qualre écluses du Rhône entre Chancy et Genève, étudiées par le Syndicat suisse, Leurs dimensions présentent une largeur de 9 mètres, une lon- 